\title{
INNOVATION RESEARCH ON STRAPDOWN INERTIAL NAVIGATION TECHNOLOGY
}

\author{
Yucui YANG*1, Yi SONG ${ }^{1}$, Yanhua ZHU2 \\ ${ }^{1}$ Huaiyin Normal University, HuaiAn, 223300 China; \\ ${ }^{2}$ Southeast University, Nanjing, 210096 China. \\ Corresponding author: Yucui YANG \\ Email: yangyucui01@126.com
}

\begin{abstract}
The independent innovation of inertial navigation system is a crucial direction and approach and means of its development, and it is targeted on the existing problems of inertial navigation system. Based on the quaternion theory which has been maturely applied in the attitude computation of strapdown inertial navigation system, this paper puts forward the innovative thinking of geometric theory and graphic theory in the field of navigation. Then, through the successful research results of the internal damping network in the strapdown system, it is necessary to fully explore the importance and practical significance of the inertial navigation potential by drawing on the platform design idea. Finally, the advices of the crucial aspects of strapdown inertial navigation system independent innovation are given.
\end{abstract}

Keywords: Inertial Navigation System; Quaternion; Geometric, Internal Damping.

\section{Introduction}

Strapdown inertial navigation system attaches inertial components directly to the carrier, and relies on computer to achieve the functions of the navigation platform. The system size, weight and cost are greatly reduced and are convenient for redundant configuration since the electromechanical navigation system is removed, which improves system performance and reliability, and meanwhile providing more navigation guidance information.

The strapdown inertial navigation system is the main trend of the future inertial navigation system development [1, 2]. But the low accuracy of strapdown inertial navigation is the major factor which constrains its development. Therefore, improving and enhancing the system's accuracy through all new ideas, new methods and new techniques is an important aspect of inertial navigation independent innovation.

This paper pays wide attention to the development and technology achievements of various current subjects, and considers applied them to the improvement of strapdown inertial navigations system.

\section{Application of Mathematical Methods in Strapdown Inertial Navigation Technology}

\subsection{Quaternion Theory}

The attitude computation is an important part of the strapdown inertial navigation system, which is related to the real-time updating of the carrier's attitude and the timely correction of the navigation coordinate system

Therefore, the accuracy of the attitude algorithm directly affects the navigation accuracy of the system.

Euler angle method and direction cosine algorithm were the two orginal methods to resolve the carrier's attitudes. However, the Euler angle method had a singular point in the differential equation when the carrier pitch angle was 90 degrees, which made it impossible to work in all attitudes. The direction cosine algorithm needed to resolve nine differential equations and the computational complexity was accordingly enormous [3]. Under the historical conditions at that time, there was an urgent need for an effective and full-scale way to solve the problem of carrier's attitudes computation. 
The quaternion theory is an ancient branch of mathematical theory. Before it was introduced into the computation of strapdown inertial navigation system (SINS), the field of application was quite small for quaternion theory, and it was not taken seriously by the majority of scholars. However, the scientific workers creatively applied it to solve the practical problems of space technology and strapdown inertial navigation system, bringing the use of quaternion to solve the attitude matrix method into being. This method not only solves the problem of differential equation degradation, but also has a small computation complexity, which can totally satisfy the SINS sampling rate and real - time requirement.

In order to reduce the rotation noncommutativity error occurred in the quaternion computation of carrier's attitude, the equivalent rotating vector algorithm is used to correct it in engineering projects. This algorithm can be divided into single sample algorithm, two-sample algorithm and three-sample algorithm according to different gyro sample number. At present, SINS widely takes quaternion algorithm and equivalent rotating vector algorithm to resolve the carrier's attitude.
The actual effect has been widely recognized by the inertial navigation industry professionals.

\subsection{Geometric Theory}

The main purpose of navigation is the location and orientation of the carrier in the plane or space, that is, to determine the parameters of the carrier in a plane or space at a given time. Then, the geometric theory or graphics theory of mathematical disciplines can be applied in the field of navigation, in order to promote the strapdown inertial navigation technology improvement and development. The following two examples canl illustrate this point.

\section{a. The Fast Locating and Orientating of a Carrier in a Certain Area}

Based on the location $\left(\lambda_{i}, \phi_{i}\right)$ of three known points in a region, the carrier (installed with SINS) can be accurately positioned and orientated. As shown in Figure 1:

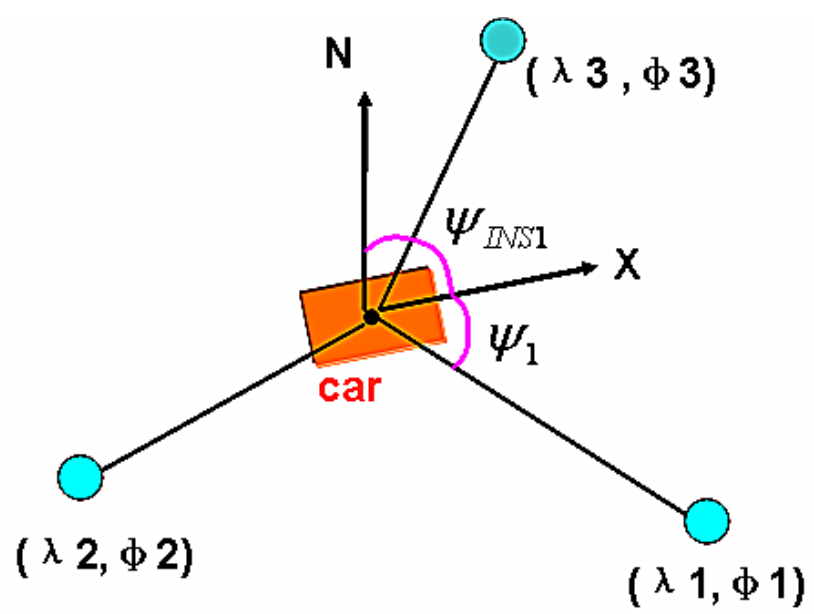

Figure 1 The Relationship Between Known Points and Car Position and Angle

SINS provides heading information $\psi_{I N S}$, The angle measuring device provides the azimuth angle of the carrier for the known point $\psi_{i}$. Then the azimuth angle of the known point relative to the north is:

$$
\psi=\psi_{\text {INSi }}+\psi_{i}
$$

And the azimuth angle of the known point relative to the north (it may be in the first quadrant) calculated according to the coordinate relation is:

$$
\psi_{c}=\arctan \frac{y_{t}-y}{x_{t}-x}
$$

Here ${ }^{x}$ and ${ }^{y}$ are the location measures output of SINS, ${ }^{x_{t}}$ and ${ }^{y_{t}}$ are known parameters, then the azimuth angle error

$$
\Delta \psi=\psi-\psi_{c}
$$

Since the inertial navigation location error is not a small amount compared to the distance between the carrier and the known point, the arctangent function cannot be linearized by the Taylor expansion method. In this case, the problem can be studied in another way: angle (heading angle) and the distance are geometric parameters, and geometric figure problem settlement can be taken into consideration. 


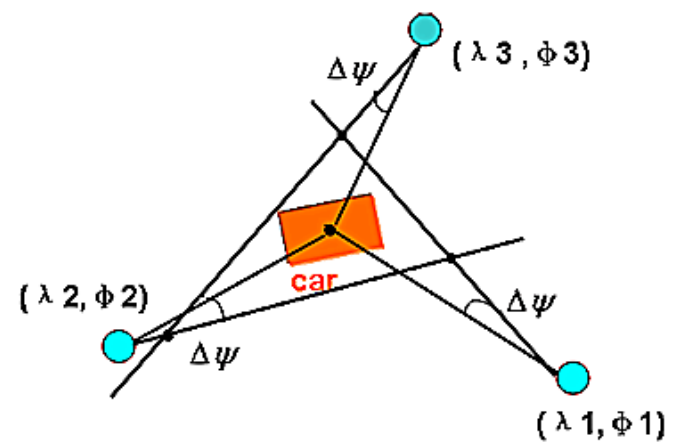

Figure 2 The Triangle Formed by Heading Angle Error

As shown in Fig. 2, assuming that the error of the azimuth angle measured by the inertia navigation is $\Delta \psi$, the location error caused by the heading angle error will form the error triangle shown in Fig. 2, and the triangular trilateral length of the triangle can be obtained mathematically. Therefore, it is not difficult to solve the problem by using the modern computer to solve the pure geometric problem, which makes the carrier position resolving problem transformed into "making side length of the error triangle be zero and minimize the position error ".

It can be seen that a complex problem in navigation can be simplified by the quick and effective geometric methods. It is known that Professor Shen Gongxun's research team from Beijing University of Aeronautics and Astronautics has embarked the research method on a carrier to quickly find the North. The key technology is the use of geometric principles to set the orientation. We can predict that the further study and refinement of the geometric model will be expected to achieve fast and accurate alignment of SINS and improve alignment and accuracy thanks to rapidity of modern computer solving mathematical problems [4, 5]. Undoubtedly, there are a lot of practical technological difficulties that need us to deep into.

\section{b. Aircraft Precise Landing}

In the aircraft landing system, the pilot plays an important role in the airport's scene judgment.

Therefore, we can consider the development of visual scene enhancing system, based on visible and infrared light detection and the use of image recognition technology to achieve the aircraft's landing alignment and monitoring on the runway [6, 7], which can provide more accurate location and orientation angle information for the pilot.

In fact, the following three graphics will be obtained through observing the aircraft runway from the viewing frame:

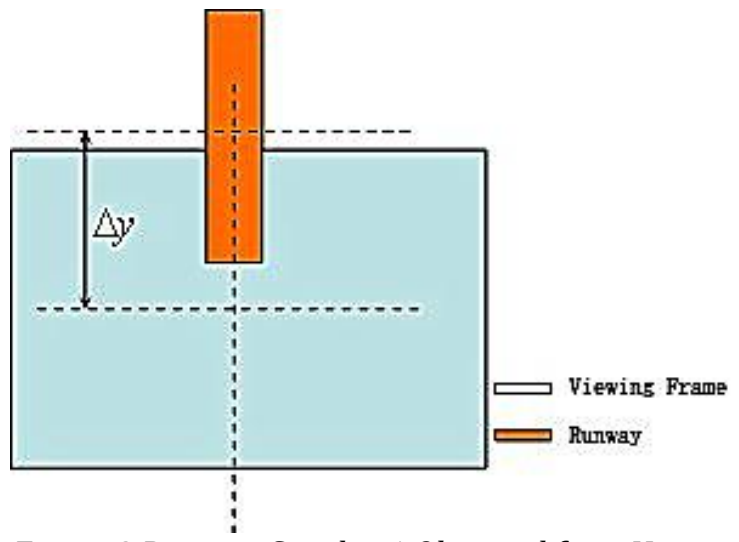

Figure 3 Runway Graphic 1 Observed from Viewing Frame

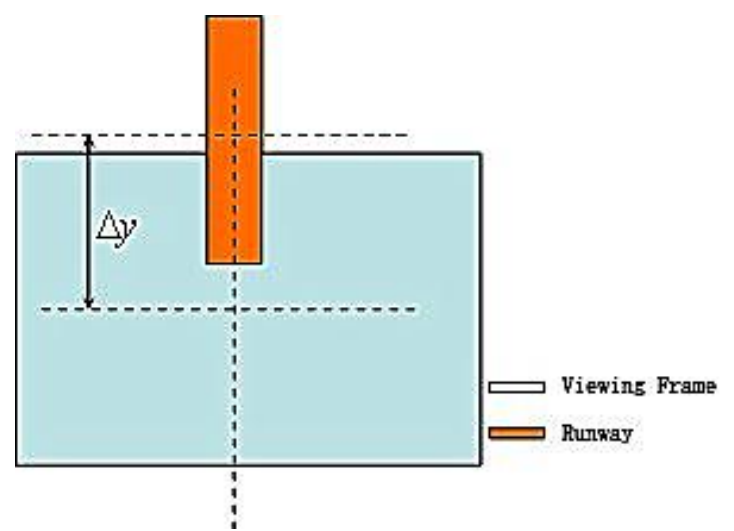

Figure 4 Runway Graphic 2 Observed from Viewing Frame

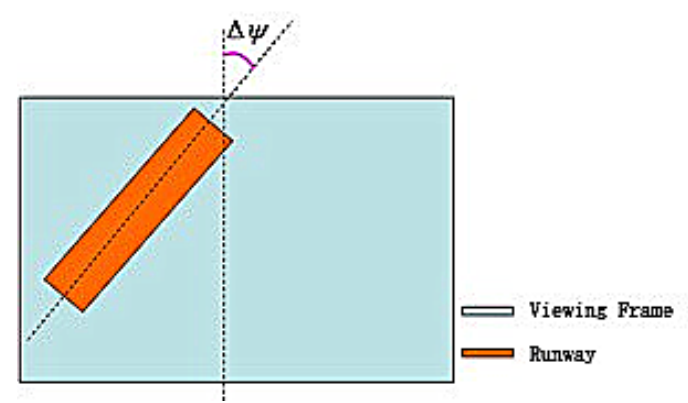

Figure 5 Runway Graphic 3 Observed from Viewing Frame 
According to the three different patterns above, three different categories of information can be acquired: Fig. 3 shows the lateral deviation; Figure 4 shows the longitudinal deviation; Figure 5 shows the azimuth angle deviation. A relatively accurate deviation value can be obtained based on the conversion of a certain proportion of graphics to guide the safe landing of the aircraft.

Undoubtedly, the above method is currently only put forward for a new research direction. The practical application still faces many technological difficulties and technical details to further research and overtake, but the concept of using geometric graphics concept is a new way to assist strapdown inertial navigation.

\section{The Use of Platform-based Internal Damping Theory in Strapdown Navigation Technology}

The existence of Schuler cycle oscillations and Fourier cycle oscillations within SINS not only generate the oscillation error, but also the accumulation error will occur due to external disturbance on SINS. This is the key factor affecting system accuracy. When the system is in the case of little acceleration, the system undamped oscillation in the traditional platform-based inertial navigation system can be effectively restrained by adding the internal damping network in the platform control circuit [8]. Other navigation information combination is usually imported in SINS to improve the navigation accuracy. We are concerned that in some special occasions, how to make full use of the inertial measurement combination of its own information to improve its accuracy when the external auxiliary sensor information is not available. This approach fully exploits the potential of inertial navigation and does not add any cost and structural complexity, which is also an important aspect of inertial navigation innovation.

This paper studies and compares the platform inertial navigation and strapdown inertial navigation fully, and refers to the design ideas of platformbased system damping network in an innovative way. The internal damping is successfully obtained in SINS, making a breakthrough. The following is a brief introduction.

The navigation system (n) adopts North East Down (NED) geographic coordinate system, the scale force equation is $f_{n}=\dot{v}_{e n}+\left(2 \omega_{i e}+\omega_{e n}\right) \times v_{e n}-g_{n}$,

The equation expansion:

$$
\left[\begin{array}{c}
f_{n x} \\
f_{n y} \\
f_{n z}
\end{array}\right]=\left[\begin{array}{c}
\dot{v}_{N}+\frac{v_{E}^{2} \tan L-v_{N} v_{D}}{R}+2 \omega_{i e} v_{E} \sin L \\
\dot{v}_{E}-\frac{v_{E}\left(v_{N} \tan L+v_{D}\right)}{R}-2 \omega_{i e}\left(v_{N} \sin L+v_{D} \cos L\right) \\
\dot{v}_{D}+\frac{v_{N}^{2}+v_{E}^{2}}{R}+2 \omega_{i e} v_{E} \cos L-g
\end{array}\right]
$$

Here, $\quad \dot{v}_{N} \dot{v}_{E} \dot{v}_{D}$ are the north accelerated speed, east accelerated speed and down accelerated speed of the system respectively; $v_{N} v_{E} v_{D}$ are the north speed, east speed and down speed respectively.; $\mathrm{L}$ is latitude; $\mathrm{R}$ is the earth radius;

$$
\left[\begin{array}{l}
f_{b x} \\
f_{b y} \\
f_{b z}
\end{array}\right]=\left[\begin{array}{ccc}
\cos \psi \cos \theta & \sin \psi \cos \theta & -\sin \theta \\
\cos \psi \sin \theta \sin \gamma-\sin \psi \cos \gamma & \sin \psi \sin \theta \sin \gamma+\cos \psi \cos \gamma & \cos \theta \sin \gamma \\
\cos \psi \sin \theta \cos \gamma+\sin \psi \sin \gamma & \sin \psi \sin \theta \cos \gamma-\cos \psi \sin \gamma & \cos \theta \cos \gamma
\end{array}\right]\left[\begin{array}{l}
f_{n x} \\
f_{n y} \\
f_{n z}
\end{array}\right]
$$

Here, $C_{n}^{b}$ is the transition matrix of the navigation coordinate system relative to the carrier coordinate system, $\gamma, \theta, \psi$ are the roll angle, pitching angle and heading angle of the carrier respectively.

When the system is in the case of little acceleration, $\dot{v}_{N}=\dot{v}_{E}=\dot{v}_{D} \approx 0$, the internal damping attitude can be calculated through the combination of the simplified equation (1), and equation (2): $\omega_{i e}$ is rotational angular velocity of the earth; $\mathrm{g}$ is the local acceleration of gravity.

The actual measurement input of accelerometer is $f_{b}$, and $f_{b}=C_{n}^{b} f_{n}$, the equation expansion is:

$$
\begin{aligned}
& \theta=\arctan \left(\frac{f_{b x}}{-f_{b z} \cos \gamma-f_{b y} \sin \gamma}\right) \\
& \gamma=\arctan \left(\frac{f_{b y}}{f_{b z}}\right)
\end{aligned}
$$

Thus, when the system is in the case of little acceleration, the attitude angle of the carrier can be calculated using the acceleration information. 
In addition, we combine the calculated attitude of strapdown inertial navigation and the inner damping attitude by Kalman filter, which can effectively suppress the oscillation of the gyro and improve the accuracy of the system. This will explained in detail due to limited space. The following model gives the prototype of the actual test results to verify the application of the attitude algorithm.

\subsection{Static Test}

The three-axis gyroscopic drift of IMU is $10^{\circ} / \mathrm{h}$, the three-axis accelerometer zero-bias is $5 \times 10^{-4} \mathrm{~g}$. Put the IMU on three-axis precision rotating table, and keep the attitude angle to be $0^{\circ}$, heading angle to be $90^{\circ}$, and do the static test for two hours.

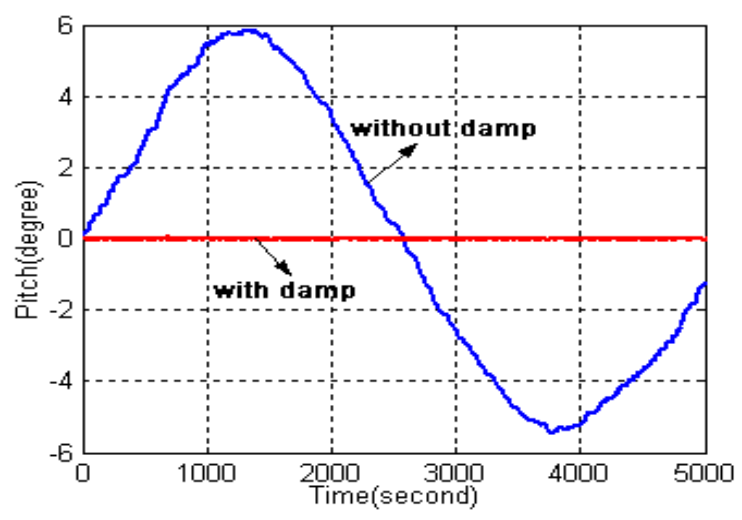

Figure 6 SINS Comparison Graphic of Static Roll Angle

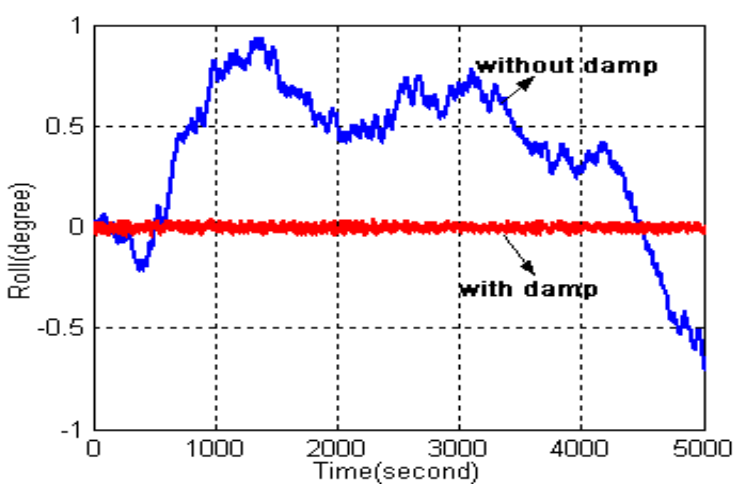

Figure 7 SINS Comparison Graphic of Static Pitching Angle

\subsection{Swing Test}

Place the IMU on the precision three-axis rotating table. Static test for more than 900 seconds, and control the rotating table around the Y-axis in the frequency of $0.15 \mathrm{~Hz}$, the amplitude of the swing movement is $\pm 30^{\circ}$, Fig. 8 and Fig. 9 are the pitching angle comparison maps of 50s time pure inertia undamped system and the internal damping system respectively.

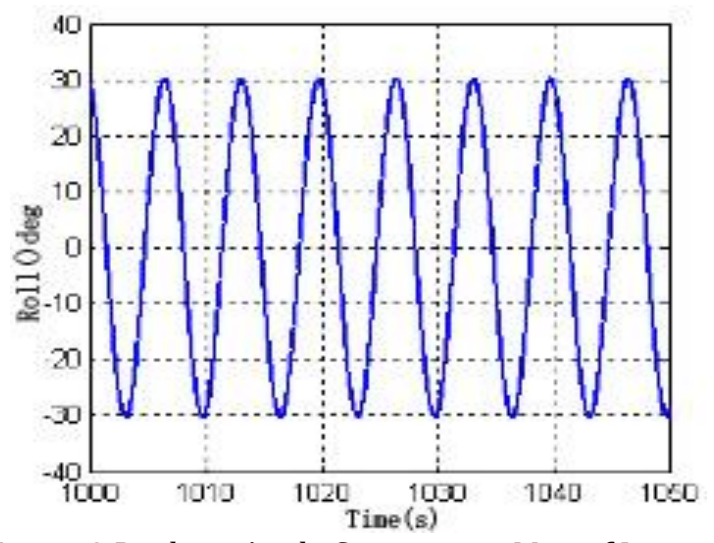

Figure 8 Pitching Angle Comparison Map of Internal Damping System

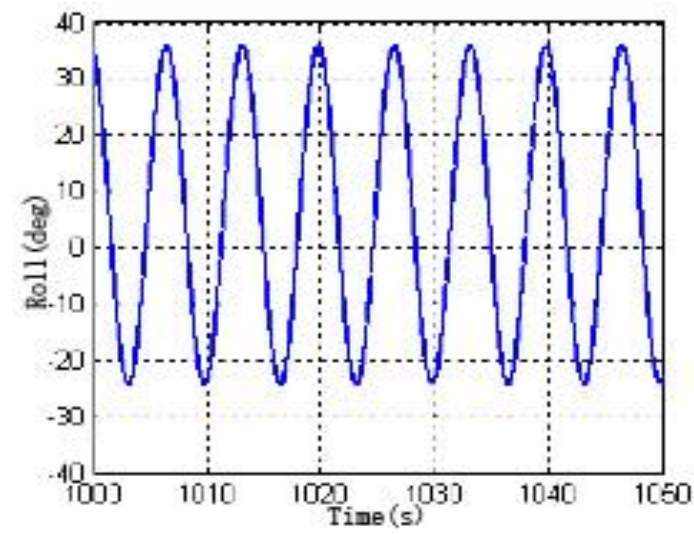

Figure 9 Pitching Angle Comparison Maps of Pure Inertia Undamped System

From the above two types of experimental results, we can see that the application of internal damping thought greatly improves the attitude accuracy of SINS. At the same time the system has also undergone a number of traffic tests, the results of the traffic tests also confirmed that the algorithm in the system greatly improved SINS accuracy in the case of little acceleration.

The research results rely on the introduction of the idea of platform inertial navigation system internal damping network in into strapdown inertial navigation. Therefore, another direction of inertial navigation system is to fully explore the ability of inertial navigation system itself as far as possible to improve its performance and accuracy.

\section{Conclusion}

The inertial navigation technology innovation and development, on the one hand depends on the further exploration and improvement of inertial navigation system itself. More importantly, it is dependent on the importation of other disciplines into the field of inertial navigation research and application, which is the important trend of the future development. 
In the following, some recommended research directions in inertial navigation systems are given.

Mathematical technology, biotechnology, image \& graphics and other disciplines are gradually emerging in the field of navigation. These technical results should be fully applied to solve the practical problems in the field of navigation;

Beacon / GPS differential combination provide users with low prices and high accuracy navigation information [9], the further research and development on beacon navigation technology will promote the progress of inertial technology;

Reasonable and reliable application of MEMS technology for small-scale inertial navigation system to make greater contributions to the relevant research $[10,11]$;

Gyro drift real-time compensation technology: computer level, modeling model and other deficiencies cannot guarantee the drift compensation be "real-time" lead to the long-term stagnation of the technology research. At present, the use of advanced computer technology and the development of theoretical results to carry out the study, the theoretical and application level of innovation are of great significance.

With the continuous efforts and innovation of our scientific researchers, these important technologies are bound to develop in the future development of inertial navigation system.

\section{Acknowledgement}

This work was supported by the 2016 National Natural Science Fund Project "Natural vector aided inertial ocean long endurance autonomous navigation technology research" (Project number: 61503077).

\section{Reference}

[1] Liu L N, Liu R Q. Research of integrated navigation system based on strapdown inertial navigation system [J]. Modern Electronics Technique, 2009, 290(3):111-113.
[2] Barbour N, Schmidt G. Inertial sensor technology trends[J]. IEEE Sensors journal, 2001, 1(4):332-339.

[3] Curey R K, Ash M E, Thielman L 0, et a1. Proposed IEEE inertial systems terminology standard and other inertial sensor standards[A]. IEEE Position Location Navigation Symposium 2004[C]. Montery, CA, 2004.

[4] Zhang C J, Yuan J N, Li D D, Wang L, Ren D L. Development trend of inertial device test technology in view of the study of accelerometer test [J]. Aerospace Control, 2005, 23(2):78-84.

[5] Azouaoui 0, Cherifi A, Bensalem R, et a1. Reinforcement learning based group navigation approach for multiple autonomous robotic system [C]. Mechatronics and Automation, 2005 IEEE International Conference, Vol. 3, 29 July-1 Aug. 2005:1539-1544.

[6] Li Z Q, Qing L F, Rong M. Study on Chariot Gun Control System Simulation in 3D-Scene [J]. Journal of System Simulation, 2006, 18 (Suppl.1): 249-251.

[7] Kim T J, Fellerhoff J R, Kohler S M. An integrated navigation system using GPS carrier phase for real-time airbore/synthetic aperture radar (SAR) [J]. Journal of the Institute of Navigation, 2001, 48(1):13-24.

[8] Du Y L, Liu J Y, Liu R H, Sun Y R. Fuzzy Damped Algorithm in Strapdown Attitude Heading Reference System [J]. Journal of Nanjing University of Aeronautics\& Astronautics, 2005, 37(3): 274-278.

[9] Lan X Q, Zhang B L, Huang J H, Huang X S. Experimental research of GPS pseudo-range differential position [J]. Journal of Hohai University (Natural Sciences), 2004, 32(3): 300303.

[10] Du Y L, Liu J Y, Li R B, Lai J Z. Strapdown Inertia Attitude Heading Reference System Based on DSP [J]. Chinese Journal of Scientific Instrument, 2005, 26(8 Suppl): 365-376.

[11] Chao H Y, Cao Y C, Chen Y Q.Autopilots for small unmanned aerial vehicles: A survey [J]. International Journal of Control, Automation and Systems, 2010, 8(1):36-44. 